TRANSACTIONS OF THE

AMERICAN MATHEMATICAL SOCIETY

Volume 277, Number 2, June 1983

\title{
SMALL INTO-ISOMORPHISMS BETWEEN SPACES OF CONTINUOUS FUNCTIONS. II
}

BY

YOAV BENYAMINI ${ }^{1}$

\begin{abstract}
We construct two compact Hausdorff spaces, $X$ and $Y$, so that $C(X)$ does not embed isometrically into $C(Y)$, but for each $\varepsilon>0$, there is an isomorphism $T_{\varepsilon}$ from $C(X)$ into $C(Y)$ satisfying $\|f\| \leqslant\left\|T_{\varepsilon} f\right\| \leqslant(1+\varepsilon)\|f\|$ for all $f \in C(X)$.
\end{abstract}

Introduction. In [2] we prove that if $X$ and $Y$ are compact Hausdorff spaces, with $X$ metrizable, then the only "small" into-isomorphisms from $C(X)$ into $C(Y)$ are the "small" perturbations of into-isometries. More precisely, if $0<\varepsilon<1$, and $T$ : $C(X) \rightarrow C(Y)$ satisfies $\|f\| \leqslant\|T f\| \leqslant(1+\varepsilon)\|f\|$ for all $f \in C(X)$, then there is an isometry $W$ from $C(X)$ into $C(Y)$ with $\|T-W\| \leqslant 3 \varepsilon$.

It was shown in [2], that this is no longer true if $X$ is not assumed to be metrizable. For every $\varepsilon>0$, we constructed two spaces $X_{\varepsilon}$ and $Y_{\varepsilon}$, with a $(1+\varepsilon)$-isomorphism $T_{\varepsilon}: C\left(X_{\varepsilon}\right) \rightarrow C\left(Y_{\varepsilon}\right)$, so that $\left\|T_{\varepsilon}-W\right\|>2$ for all isometries $W$ from $C\left(X_{\varepsilon}\right)$ into $C\left(Y_{\varepsilon}\right)$.

The purpose of this note is to give a much stronger counterexample. We construct two spaces $X$ and $Y$, so that for every $\varepsilon>0$, there is an isomorphism $T_{\varepsilon}: C(X) \rightarrow$ $C(Y)$ satisfying $\|f\| \leqslant\left\|T_{\varepsilon} f\right\| \leqslant(1+\varepsilon)\|f\|$ for all $f \in C(X)$. Yet there is no isometry whatsoever from $C(X)$ into $C(Y)$.

The idea of the construction goes as follows: For a fixed $n \geqslant 1$, we start with a pair of spaces $B \subset B_{n}$ so that there is a $(1+1 / n)$-simultaneous extension operator from $C(B)$ into $C\left(B_{n}\right)$-but not of strictly smaller norm. Next we embed $B_{n}$ in a space $Y_{n}$ so that $X_{n}=Y_{n} \backslash\left(B_{n} \backslash B\right)$ is closed, and there is a natural $(1+1 / n)$ simultaneous extension operator from $C\left(X_{n}\right)$ into $C\left(Y_{n}\right)$, i.e. a $(1+1 / n)$ isomorphism of $C\left(X_{n}\right)$ into $C\left(Y_{n}\right)$. The construction $Y_{n}$ is done so that the "relative position" of $B$ in $Y_{n}$ is very rigid: Every isometry of $C\left(X_{n}\right)$ into $C\left(Y_{n}\right)$ will-essentially_induce a norm one simultaneous extension operator from $C(B)$ into $C\left(B_{n}\right)$, which is impossible. This shows that there is no isometry from $C\left(X_{n}\right)$ into $C\left(Y_{n}\right)$, and the final example is obtained by taking the one-point-compactifications of disjoint copies of the $X_{n}$ 's and $Y_{n}$ 's, respectively.

The construction of $Y_{n}$ uses the existence of an uncountable family of strongly noncomparable compact Hausdorff spaces. For the sake of completeness we describe such a construction (due to R. Bonnet [5]) in $\$ 4$.

Received by the editors July 22, 1982.

1980 Mathematics Subject Classification. Primary 46E15.

'This research was partially supported by the Fund for the Promotion of Research at the TechnionIsrael Institute of Technology. 
Our notation and terminology are standard. All topological spaces will be Hausdorff, and all operators will be linear and bounded. We treat the real case only-hardly any change is needed for the complex case.

ACKNOWLEDGEMENT. It is a pleasure to thank M. Rubin for the detailed references and description of rigid and uncomparable spaces. We also thank S. Shelah for clarifying for us the construction in $\S 4$ when $\mathrm{CH}$ is not assumed.

1. Preliminaries. We start with a well-known description of into-isometries between spaces of continuous functions.

Lemma 1. Let $X$ and $Y$ be two compact spaces so that $C(X)$ embeds isometrically into $C(Y)$. Then there is a closed subset $F$ of $Y$, a continuous map $\varphi$ from $F$ onto $X$, and an isometry $W$ of $C(X)$ into $C(Y)$, so that $(W f)(y)=f(\varphi(y))$ for all $y \in F$ and $f \in C(X)$.

Proof. Let $U$ be an isometry of $C(X)$ into $C(Y)$, and put $\varepsilon(y)=(U 1)(y)$ (where 1 is the function identically 1 on $X$ ). It is well known (see e.g. [8]), that there is a closed subset $F$ of $Y$, and a continuous map $\varphi$ from $F$ onto $X$, so that $|\varepsilon(y)|=1$ for $y \in F$, and $(U f)(y)=\varepsilon(y) f(\varphi(y))$ for $y \in F$ and $f \in C(X)$. The isometry $W$ is defined by $(W f)(y)=\varepsilon(y)(U f)(y)$ for all $y \in Y$ and $f \in C(X)$.

The next two lemmas summarize the properties of the spaces $B, B_{n}$ and the strongly noncomparable family $K_{\alpha}$ which will be needed in the construction. The spaces $B$ and $B_{n}$ will be described in $\S 3$, and the $K_{\alpha}$ 's in $\S 4$.

We first recall some definitions and notation. By $\omega_{1}$ we denote the first uncountable ordinal, as well as its cardinality. We denote by $\mathscr{D}$ the one-point-compactification of a discrete set of cardinality $\omega_{1}$.

A compact space is called CCC, if every collection of mutually disjoint open sets is at most countable.

If $K$ is a compact space, and $H$ is a closed subset of $K$, then a simultaneous extension operator (SEO) from $C(H)$ into $C(K)$, is a bounded linear operator $S$ : $C(H) \rightarrow C(K)$ so that $\left.S f\right|_{H}=f$ for all $f \in C(H)$.

LEMMA 2. There is a compact space $B$, and for each $n=1,2, \ldots$ a compact space $B_{n}$ containing $B$, so that:

(i) There is a SEO $S_{n}: C(B) \rightarrow C\left(B_{n}\right)$ with $\left\|S_{n}\right\|=1+1 / n$.

(ii) Every relatively open subset of $B$ contains a compact subset $H$, which is a retract of $B$, and so that if $S: C(H) \rightarrow C\left(B_{n}\right)$ is $S E O$ then $\|S\| \geqslant 1+1 / n$.

(iii) If $K$ is a (Hausdorff) continuous image of a closed subset of $B_{n}$, and if $K$ is $C C C$, then $K$ is metrizable;

(iv) There is a dense subset $\left\{b_{\alpha}: \alpha<\omega_{1}\right\}$ of $B$, so that every uncountable subset of the $b_{\alpha}$ 's contains a further subset whose closure in $B$ is homeomorphic to $\mathscr{D}$.

LEMMA 3. There is an uncountable family $\left\{K_{\alpha}: \alpha<\omega_{1}\right\}$ of compact spaces, so that:

(i) The different $K_{\alpha}$ 's are strongly noncomparable: if $S$ is a closed subset of $K_{\alpha^{\prime}}$ and $\varphi$ is a continuous map from $S$ into $K_{\beta}$ so that $\varphi(S)$ has nonempty interior, then necessarily $\alpha=\beta$. 
(ii) Each $K_{\alpha}$ is hereditarily CCC, i.e. every closed subset of $K_{\alpha}$ is CCC. (In particular $K_{\alpha}$ does not contain a subspace homeomorphic to .).)

(iii) Every closed metrizable subset of $K_{\alpha}$ has empty interior.

2. The example. Let $B, B_{n},\left\{b_{\alpha}\right\}$ and $\left\{K_{\alpha}\right\}$ be as in Lemmas 2 and 3 . We construct the space $Y_{n}$ by "replacing" each of the points $b_{\alpha}$ in $B \subseteq B_{n}$ by the space $K_{\alpha}$. To make this precise, choose for each $\alpha$ a point $\bar{b}_{\alpha} \in K_{\alpha}$, and let $Y_{n}$ be the set obtained from the disjoint union $B_{n} \cup\left(\cup_{\alpha} K_{\alpha}\right)$ by identifying each point $b_{\alpha} \in B$ with the point $\bar{b}_{\alpha} \in K_{\alpha}$. (Thus from now on we shall not distinguish between $b_{\alpha}$ and $\bar{b}_{\alpha}$, and we shall use the same notation, $b_{\alpha}$, for both.)

The following sets will constitute a subbase for the topology on $Y_{n}$ :

For each fixed $\alpha$, the open subsets $U$ of $K_{\alpha}$ so that $b_{\alpha} \notin U$.

For each fixed $\alpha$, the sets of the form $V \cup\left\{\cup\left\{K_{\beta}: b_{\beta} \in V, \beta \neq \alpha\right\}\right\} \cup U$, where $V$ is an open subset of $B_{n}$ and $U$ an open subset of $K_{\alpha}$, containing $b_{\alpha}$.

It is easy to check that this defines a compact Hausdorff topology on $Y_{n}$, which gives the original topology on $B_{n}$ and on each of the $K_{\alpha}$ 's. Moreover, a net $\left(y_{\nu}\right)$ converges to a point $y$, where $y_{\nu} \in K_{\alpha_{\nu}}$ and $\alpha_{\nu} \neq \alpha_{\mu}$ iff $y \in B$ and $b_{\alpha_{v}} \rightarrow y$. (This topology can be realized as a "porcupine topology" as defined in [4].)

It follows that $B_{n} \backslash B$ is an open subset of $Y_{n}$, and we define $X_{n}=Y_{n} \backslash\left(B_{n} \backslash B\right)$. The set $X_{n}$ is a closed subset of $Y_{n}$, and since the $K_{\alpha}$ 's were "glued" to $B_{n}$ at points of $B$, the spaces $X_{n}$, for different $n$, are mutually homeomorphic.

The following lemma summarizes some simple properties of $X_{n}$ and $Y_{n}$.

LEMMA 4. With $X_{n}$ and $Y_{n}$ as above we have:

(a) There is a SEO, $T_{n}: C\left(X_{n}\right) \rightarrow C\left(Y_{n}\right)$ with $\left\|T_{n}\right\|=1+1 / n$.

(b) Every relatively open subset $V$ of $B$, contains a compact subset $H$ so that

(b-i) there is a norm one SEO from $C(H)$ into $C\left(X_{n}\right)$ and

(b-ii) there is no norm one SEO from $C(H)$ into $C\left(Y_{n}\right)$.

Proof. (a) Let $S_{n}: C(B) \rightarrow C\left(B_{n}\right)$ be the SEO given by Lemma 2(i). Define $T_{n}$ : $C\left(X_{n}\right) \rightarrow C\left(Y_{n}\right)$ by

$$
(T f)(y)= \begin{cases}f(y), & y \in X_{n}, \\ S_{n}\left(\left.f\right|_{B}\right)(y), & y \in B_{n} \backslash B .\end{cases}
$$

It is easy to check that $T f \in C\left(Y_{n}\right)$ and extends $f$, and that $\left\|T_{n}\right\|=\left\|S_{n}\right\|=1+$ $1 / n$.

(b) Let $H$ be the subset of $V$ given by Lemma 2(ii). It is easy to check that the map

$$
r(x)= \begin{cases}x, & x \in B, \\ b_{\alpha}, & x \in K_{\alpha},\end{cases}
$$

is a retraction of $X_{n}$ onto $B$. Since $H$ is a retract $B$, it follows that there is a retraction $q$ from $X_{n}$ onto $H$. Setting $(S f)(x)=f(q(x))$ gives a norm one SEO from $C(H)$ into $C\left(X_{n}\right)$, thus proving (b-i).

To prove (b-ii), assume $T: C(H) \rightarrow C\left(Y_{n}\right)$ is a SEO, and let $R: C\left(Y_{n}\right) \rightarrow C\left(B_{n}\right)$ be the restriction operator. It follows that $R T: C(H) \rightarrow C\left(B_{n}\right)$ is a SEO, and by Lemma 2(ii), $\|R T\| \geqslant 1+1 / n$. Since $\|R\|=1$ it follows that $\|T\| \geqslant 1+1 / n>1$. 
The next lemma contains the main idea of our construction. We first need some notation. Since all the spaces $X_{n}$ are mutually homeomorphic, and can be naturally identified, we let $Z$ be the space homeomorphic to the $X_{n}$ 's, and we put $\psi_{n}: Z \rightarrow X_{n}$ to be the natural homeomorphism. We shall also want to distinguish between the copies of $K_{\alpha}$ in the different $Y_{n}$ 's, so we denote by $K(\alpha, n)$ the copy of the space $K_{\alpha}$ in $Y_{n}$. Let $Y$ be the one-point-compactification of the disjoint union of the $Y_{n}$ 's, and let $p$ be the point of infinity.

LeMma 5. Let $F$ be a closed subset of $Y$, and let $\varphi$ be a continuous map from $F$ onto $Z$. Then there is an $N$ and a relatively open subset $V$ of $B$, so that $\psi_{N}(V) \subset F$, and so that $\varphi\left(\psi_{N}(v)\right)=v$ for all $v \in V$. In particular, $\varphi$ is one-one on $\psi_{N}(V)$ and is a homeomorphism on $\psi_{N}(H)$ for any compact subset $H$ of $V$.

Proof. Let $U$ be an open subset of $Z$ so that $U \cap B \neq \varnothing$ and so that $\varphi(p) \notin \bar{U}$. (If $p \notin F$ we can just take $U=Z$.) Since $\varphi(p) \notin \bar{U}$, there is an $M$ so that $\varphi^{-1}(U) \subset \cup_{n=1}^{M} Y_{n}$.

Fix now any $\alpha$ so that $b_{\alpha} \in U \cap B$. We shall prove the following claim:

There is an $1 \leqslant n \leqslant M$ so that $F \cap K(\alpha, n) \neq \varnothing$, and a point $s_{\alpha} \in F \cap K(\alpha, n)$ so that $\varphi\left(s_{\alpha}\right) \in K_{\alpha}$.

Once the claim is proved, the lemma follows. Indeed, fix any point $b \in B \cap U$ and find a net $b_{\alpha_{\nu}}$ of different points in $B \cap U$ converging to $b$. (This is possible, because $\left\{b_{\alpha}\right\}$ is dense in $B$.) Let $s_{\alpha_{\nu}}$ be the points guaranteed by the claim, and by passing to a subnet if necessary, we can assume that there is a $1 \leqslant n_{0} \leqslant M$, so that $s_{\alpha_{\nu}} \in F \cap K\left(\alpha_{\nu}, n_{0}\right)$ for all $\nu$. By the definition of the topology on $Y_{n}$ and $Z$, it follows from $b_{\alpha_{\nu}} \rightarrow b$ and the fact that $s_{\alpha_{\nu}} \in K\left(\alpha_{\nu}, n_{0}\right)$ and $\varphi\left(s_{\alpha_{\nu}}\right) \in K_{\alpha_{\nu}}$, that we also have $s_{\alpha_{\nu}} \rightarrow \psi_{n_{0}}(b)$, and $\varphi\left(s_{\alpha_{\nu}}\right) \rightarrow b$. Since $F$ is closed $s_{\alpha_{v}} \rightarrow \psi_{n_{0}}(b)$ implies that $\psi_{n_{0}}(b) \in F$, and then the continuity of $\varphi$ gives that $\varphi\left(\psi_{n_{0}}(b)\right)=b$.

If we put, for $n \leqslant M, A_{n}=\left\{b \in B: \psi_{n}(b) \in F\right.$ and $\left.\varphi\left(\psi_{n}(b)\right)=b\right\}$ the above argument shows that the claim implies that $U \cap B \subset \cup_{n=1}^{M} A_{n}$. Since each $A_{n}$ is closed, it follows from the Baire category theorem that one of the $A_{n}$ 's, say $A_{N}$, has nonempty interior in $B$, which is exactly what we had to prove.

We now prove the claim.

Fix any $\alpha$ so that $b_{\alpha} \in U \cap B$. We shall show that there is a $\beta$ and $n \leqslant M$, so that $F \cap K(\beta, n) \neq \varnothing$, and so that $\varphi(F \cap K(\beta, n))$ has nonempty interior in $K_{\alpha}$. By the strong noncomparability of the different $K$ 's (Lemma 3(i)), it follows that necessarily $\beta=\alpha$, proving the claim.

Now $K_{\alpha}$ is hereditarily CCC, and thus for each $n, \varphi\left(F \cap B_{n}\right) \cap K_{\alpha}$ is CCC, hence metrizable (by Lemma 2(iii)). Lemma 3(iii) now implies that $K_{\alpha} \cap\left(\cup_{n} \varphi\left(F \cap B_{n}\right)\right)$ is of first category in $K_{\alpha}$.

Assuming, for contradiction, that $\varphi(F \cap K(\beta, n)) \cap K_{\alpha}$ has empty interior in $K_{\alpha}$ for all $\beta$ and all $n \leqslant M$, we will proceed by transfinite induction to find an uncountable set $A \subset \omega_{1}$, and for each $\beta \in A$ an $n \leqslant M$ and a point $s_{\beta} \in F \cap K(\beta, n)$ with $\varphi\left(s_{\beta}\right) \in K_{\alpha}$ and $\varphi\left(s_{\beta}\right) \neq \varphi\left(s_{\gamma}\right)$ for all $\beta \neq \gamma$ in $A$.

Indeed, since $\left(\cup \varphi\left(F \cap B_{n}\right)\right) \cap K_{\alpha}$ is of first category in $K_{\alpha}$, while

$$
\varphi(F) \supset K_{\alpha} \cap U,
$$


there is a $\beta_{1}$ and $n \leqslant M$ so that $K\left(\beta_{1}, n\right) \cap F \neq \varnothing$ and a point $s_{\beta_{1}} \in K\left(\beta_{1}, n\right) \cap F$ so that $\varphi\left(s_{\beta_{1}}\right) \in K_{\alpha} \backslash \cup_{n} \varphi\left(F \cap B_{n}\right)$.

Inductively, having already chosen a countable subset $A_{0} \subset \omega_{1}$ and points $s_{\beta} \in$ $K\left(\beta, n_{\beta}\right) \cap F$ for $\beta \in \mathrm{A}_{0}$, the union

$$
\left(\bigcup_{\substack{\beta \in A_{0} \\ n \leqslant M}} \varphi(F \cap K(\beta, n))\right) \cup\left(\bigcup_{n} \varphi\left(F \cap B_{n}\right)\right)
$$

is of first category in $K_{\alpha}$, and $\varphi(F) \supset K_{\alpha} \cap U$; thus there is a $\beta \notin A_{0}, n \leqslant M$ and a point $s_{\beta} \in F \cap K(\beta, n)$, so that $\varphi\left(s_{\beta}\right) \in K_{\alpha}$, but $\varphi\left(s_{\beta}\right)$ is not in the above union. This defines $A$. In particular $\varphi\left(s_{\beta}\right) \neq \varphi\left(s_{\gamma}\right)$ for all $\gamma \neq \beta$ in $A$, and since $A$ is uncountable, we can assume that there is a fixed $n_{0}$, so that $s_{\beta} \in K\left(\beta, n_{0}\right)$ for all $\beta \in A$.

By Lemma 2(iv) there is an uncountable subset $A^{\prime}$ of $A$, so that the closure of $\left\{\psi_{n_{0}}\left(b_{\beta}\right): \beta \in A^{\prime}\right\}$ in $\psi_{n_{0}}(B)$ is homeomorphic to $\mathscr{D}$. The topology on $Y_{n_{0}}$ then implies that also the closure of $\left\{s_{\beta}: \beta \in A^{\prime}\right\}$ is homeomorphic to $\mathscr{D}$. But $\varphi$ is one-one on $\left\{s_{\beta}\right.$ : $\left.\beta \in A^{\prime}\right\}$, and thus the closure of $\left\{\varphi\left(s_{\beta}\right): \beta \in A^{\prime}\right\}$ in $K_{\alpha}$ is also homeomorphic to $\mathscr{D}$, and this contradicts Lemma 3(ii). Thus such an uncountable procedure is impossible, and there is a $\beta$ and $n$ so that $\varphi(K(\beta, n) \cap F)$ has nonempty interior in $K_{\alpha}$. This implies the claim, and hence the lemma.

We are now ready for the example. The space $Y$ was already defined, and we take $X$ to be the one-point-compactification of a countable disjoint family of copies of $Z$.

ThEOREM. $C(X)$ does not embed isometrically into $C(Y)$ but, for every $\varepsilon>0$, there is an isomorphism $T: C(X) \rightarrow C(Y)$ satisfying $\|f\| \leqslant\|T f\| \leqslant(1+\varepsilon)\|f\|$ for all $f \in C(X)$.

Proof. Fix any $\varepsilon>0$. We first construct a $(1+\varepsilon)$-isomorphism from $C(X)$ into $C(Y)$. Indeed, fix $N>1 / \varepsilon$, and identify $X$ as the closed subset $\{p\} \cup\left(\cup_{n \geqslant N} X_{n}\right)$ in $Y$. Let $T_{n}: C\left(X_{n}\right) \rightarrow C\left(Y_{n}\right)$ be the SEO given by Lemma 4(a), and define $T$ : $C\left(\{p\} \cup\left(\cup_{n \geqslant N} X_{n}\right)\right) \rightarrow C(Y)$ by

$$
(T f)(y)= \begin{cases}0, & y \in \cup_{n<N} Y_{n}, \\ f(p), & y=p, \\ T_{n}\left(\left.f\right|_{X_{n}}\right)(y) & y \in Y_{n}, n \geqslant N .\end{cases}
$$

It is easy to check that $T$ is a SEO of norm $1+1 / N<1+\varepsilon$, and thus a $(1+\varepsilon)$-isomorphism of $C(X)$ into $C(Y)$.

To prove the other part of the theorem, we show an even stronger result: There is no isometry from $C(Z)$ into $C(Y)$.

Indeed, assume such an isometry exists, and find, using Lemma 1, a closed subset $F$ of $Y$, a continuous map $\varphi$ from $F$ onto $Z$, and an isometry $W$ from $C(Z)$ into $C(Y)$ so that $W f(y)=f(\varphi(y))$ for all $y \in F$.

By Lemma 5 , there is a relatively open subset $V$ of $B$, and an $N$, so that $\psi_{N}(V) \subset F$, and so that $\varphi\left(\psi_{N}(v)\right)=v$ for all $v \in V$. 
To save cumbersome notation, we shall now identify $Z$ with $X_{N}$. (I.e. $\psi_{N}$ will be the identity map and $\varphi(v)=v$ for all $v \in V$. The isometry $W$ will be considered as an operator from $C\left(X_{N}\right)$ into $C(Y)$.)

Let $H \subset V$ be as in Lemma 4(b), and let $S_{N}: C(H) \rightarrow C\left(X_{N}\right)$ be a norm one SEO. Let $R_{N}: C(Y) \rightarrow C\left(Y_{N}\right)$ be the restriction operator; then $\left\|R_{N} W S_{N}\right\|=1$. Moreover, if $f \in C(H)$, and $y \in H$ then $R_{N} W S_{N} f(y)=y$ (because then $y \in V$ and $\varphi(y)=y$ on $V$ ), i.e. $R_{N} W S_{N}$ is a norm one SEO from $C(H)$ into $C\left(Y_{N}\right)$, which is impossible by Lemma $4(\mathrm{~b}-\mathrm{ii})$.

3. The spaces $B$ and $B_{n}$. Let $B$ be the unit ball of a Hilbert space, say $l_{2}(\Gamma)$, where $\Gamma$ has cardinality $\omega_{1}$. Let $\left\{e_{\gamma}: \gamma<\omega_{1}\right\}$ be the unit vector basis for $l_{2}(\Gamma)$. Equipped with the weak topology, $B$ is a compact Hausdorff space.

Lemma 6. Let $V$ be an open subset of $B$. Then $V$ contains a compact subset $H$, homeomorphic to $B$, which is a retract of $B$.

Proof. Fix $v \in V$ with $\|v\|<1$. There exists a finite subset $\Gamma_{0}$ of $\Gamma$ so that $H=\left\{b \in B: b(\gamma)=v(\gamma)\right.$ for all $\left.\gamma \in \Gamma_{0}\right\}$ is contained in $V$ (because $V$ is weakly open). Also, it follows from $\|v\|<1$ that $H$ is homeomorphic to $B$.

To define a retraction from $B$ onto $H$, put $a=\left(\Sigma_{\Gamma_{0}}|v(\gamma)|^{2}\right)^{1 / 2}$ and let $f:[0,1] \rightarrow[0,1]$ be a continuous function so that $f(a)=1$ and $a^{2}+\left(1-t^{2}\right) f^{2}(t) \leqslant$ 1 for all $0 \leqslant t \leqslant 1$. Define now $r: B \rightarrow H$ by

$$
r(b)(\gamma)= \begin{cases}v(\gamma), & \gamma \in \Gamma_{0}, \\ f\left(\left(\Sigma_{\Gamma_{0}}|b(\beta)|^{2}\right)^{1 / 2}\right) b(\gamma), & \gamma \notin \Gamma_{0} .\end{cases}
$$

It is clear that $r$ is continuous, and that $r(b)=b$ for $b \in H$ (because $f(a)=1$ ). Also if $b \in B$, put $t=\left(\Sigma_{\Gamma_{0}}|b(\beta)|^{2}\right)^{1 / 2}$, then

$$
\|r(b)\|^{2}=a^{2}+f^{2}(t)\left(\sum_{\beta \notin \Gamma_{0}}|b(\beta)|^{2}\right) \leqslant a^{2}+f^{2}(t)\left(1-t^{2}\right) \leqslant 1,
$$

and thus $r(b) \in H$ for all $b \in B$.

We identify $C(B)^{*}$ with the space of regular Borel measures of $B$. For each $n$, put $B_{n}=\left\{\mu \in C(B)^{*}:\|\mu\| \leqslant 1+1 / n\right\}$. Equipped with the $\omega^{*}$-topology, $B_{n}$ is a compact Hausdorff space, and we identify $B$ as a closed subset of $B_{n}$ by considering every $b \in B$ as the unit-mass measure concentrated at $b$.

Proof of Lemma 2. (i) Define $S_{n}$ by the formula $\left(S_{n} f\right)(\mu)=\int f d \mu$ for all $f \in C(B)$ and $\mu \in B_{n}$.

(ii) Let $V$ be a relatively open subset of $B$, and let $H \subset V$ be as in Lemma 6. Let $\Sigma_{n}$ be the set of all measures in $B_{n}$ whose support is contained in $H$. $\Sigma_{n}$ is a closed subset of $B_{n}$, and it can be identified with $\left\{\mu \in C(H)^{*}:\|\mu\| \leqslant 1+1 / n\right\}$.

It was proved in [1] that every SEO $T: C(B) \rightarrow C\left(B_{n}\right)$ satisfies $\|T\| \geqslant 1+1 / n$. Since $H$ is homeomorphic to $B$ it follows that every SEO $T: C(H) \rightarrow C\left(\Sigma_{n}\right)$, satisfies $\|T\| \geqslant 1+1 / n$. Let now $S: C(H) \rightarrow C\left(B_{n}\right)$ be a SEO, and let $R: C\left(B_{n}\right) \rightarrow C\left(\Sigma_{n}\right)$ be the restriction operator. Then $R S: C(H) \rightarrow C\left(\Sigma_{n}\right)$ is a SEO, and it follows that $1+1 / n \leqslant\|R S\| \leqslant\|S\|$. 
(iii) The space $B_{n}$ is an Eberlein compact, and by [3] a continuous image of an Eberlein compact is itself an Eberlein compact. It is well known (see e.g. [3]) that a CCC Eberlein compact is metrizable.

(iv) The set $\left\{b_{\alpha}: \alpha<\omega_{1}\right\}$ of all vectors in $B$ with finite support and rational coordinates is a dense subset of $B$ with cardinality $\omega_{1}$. Given any uncountable family $\left\{b_{\alpha}: \alpha \in A\right\}$ of these, there is an uncountable subset $A^{\prime} \subset A$, so that the supports of $\left\{b_{\alpha}: \alpha \in A^{\prime}\right\}$ are quasidisjoint, i.e. there is a finite subset $\Gamma_{0}$ of $\Gamma$ so that for each $\gamma \notin \Gamma_{0}$, there is at most one $\alpha \in A^{\prime}$ with $b_{\alpha}(\gamma) \neq 0$ (see e.g. [7, p. 87]).

Since $\Gamma_{0}$ is finite, and the coordinates of the $b_{\alpha}$ 's are rational, it follows that the uncountable set $A^{\prime}$ has a further uncountable subset $A^{\prime \prime}$ so that there is a fixed vector $v$, supported in $\Gamma_{0}$, and such that $\left.b_{\alpha}\right|_{\Gamma_{0}}=\left.v\right|_{\Gamma_{0}}$ for all $\alpha \in A^{\prime \prime}$. But it now follows that the closure of $\left\{b_{\alpha}: \alpha \in A^{\prime \prime}\right\}$ is exactly $\{v\} \cup\left\{b_{\alpha}: \alpha \in A^{\prime \prime}\right\}$, and it is homeomorphic to $\mathscr{Q}$.

4. The spaces $K_{\alpha}$. For the sake of completeness, we reproduce in this section, a construction due to $\mathrm{R}$. Bonnet [5] of a family of strongly noncomparable spaces. To avoid inessential technical details, we present the construction under the Continuum Hypothesis. In the general case, the only essential modification is needed in Lemma 8, where the condition that $\{q \in Q: f(q) \neq q\}$ is at most countable, is replaced by the condition that its cardinality is less than that of the Continuum. We refer the reader to [5] for the details.

The spaces $K_{\alpha}$ are constructed as the Stone spaces of certain Boolean algebras. The reader is referred to [6] for the properties of Boolean algebras and their Stone spaces which we use freely. (We use, however, the term Stone space, rather than Boolean space as in [6].)

Let $P$ be a subset of $\mathbf{R}$, and denote, for $a, b \in P$, the set $\{p \in P: a \leqslant p<b\}$ by $[a, b)$. We also allow $a=-\infty$ or $b=+\infty$, where we understand $[-\infty, b)$ to be $\{p \in P:-\infty<p<b\}$.

Let $B(P)$ be the Boolean algebra of all subsets of $P$ of the form $\cup_{i=1}^{m}\left[a_{2 i-1}, a_{2 i}\right)$ for $a_{1}<a_{2}<\cdots<a_{2 m}$ in $P$. (We also allow $a_{1}=-\infty, a_{2 m}=+\infty$.) We denote by $K(P)$ the Stone space of $B(P)$. The space $K(P)$ is compact, Hausdorff and totally disconnected. Its clopen sets can be identified with the elements of $B(P)$.

LEMMA 7. Every closed subset of $K(P)$ is a retract of $K(P)$.

Proof. Let $S$ be a closed subset of $K(P)$, and identify it as the Stone space of $B=B(P) / J$ for some ideal $J$ in $B(P)$. Let $\pi: B(P) \rightarrow B$ be the quotient map. The set $Q=\{\pi(-\infty, p): p \in P\}$ is a set of pairwise comparable generators for $B$. Let $\sigma$ : $Q \rightarrow B(P)$ be an increasing one-one map, so that $\pi(\sigma(q))=q$ for all $q \in Q$. Using the fact that elements in $Q$ are pairwise comparable and that $\sigma$ is increasing, it follows that $\sigma$ extends to an isomorphism (still devoted by $\sigma$ ) of $B$ into $B(P)$, so that $\pi(\sigma(b))=b$ for all $b \in B$. The isomorphism $\sigma$ is induced by a continuous map $\varphi$ from $K(P)$ onto $S$, and the condition $\pi \sigma(b)=b$ for all $b \in B$ implies that $\varphi$ is a retraction.

The main feature of the spaces $K(P)$ is that the order structure of the set $P$ is reflected in its topology. In order to use this phenomena, we shall need the following 
rather surprising lemma. (This lemma of R. Bonnet generalizes an earlier result of W. Sierpinsky [9].)

Lemma 8. Assume the Continuum Hypothesis. Then there exists an uncountable subset $P$ of $\mathbf{R}$, such that if $Q$ is any subset of $P$ and $f: Q \rightarrow P$ is any strictly monotone function (decreasing or increasing) then $\{q \in Q: f(q) \neq q\}$ is at most countable.

Proof. For each strictly monotone function $f$ defined on a subset of $\mathbf{R}$, let $G(f)$ denote the closure of the graph of $f$. Since there are only $\omega_{1}$ closed subsets of $\mathbf{R}^{2}$, we can write all these "closed graph" as a transfinite sequence $\left\{G_{\alpha}: \alpha<\omega_{1}\right\}$. If $G=G(f)$ is such a "closed graph", it need not be a graph of a function, and even if it is, it need not be a graph of a strictly monotone function. But it "almost" is such a graph: There are at most countably many values of $x$ such that there are $y \neq z$ so that either $(x, y)$ and $(x, z)$ are both in $G$, or $(y, x)$ and $(z, x)$ are both in $G$. For each $\alpha<\omega_{1}$, denote by $A_{\alpha}$ this countable set of exceptional points for $G_{\alpha}$.

We now define $P$ by transfinite induction: We choose $p_{1}$ arbitrarily. Having chosen $\left\{p_{\beta}: \beta<\alpha\right\}$, we choose $p_{\alpha}$ in the complement of the countable set

$$
\begin{aligned}
\left\{p_{\beta}: \beta<\alpha\right\} & \cup\left(\cup\left\{A_{\beta}: \beta \leqslant \alpha\right\}\right) \\
& \cup\left\{x: \exists \beta, \gamma<\alpha \text { with }\left(p_{\beta}, x\right) \in G_{\gamma}, \text { or }\left(x, p_{\beta}\right) \in G_{\gamma}\right\} .
\end{aligned}
$$

Assume now that $Q \subset P$ is uncountable, and $f: Q \rightarrow P$ is strictly monotone, and find $\alpha$ so that $G_{\alpha}=G(f)$. Let $Q_{1} \subset Q$ be the countable set $\left\{q \in Q: q=p_{\beta}\right.$ or $f(q)=p_{\beta}$ for some $\left.\beta \leqslant \alpha\right\}$. We shall show that $f(q)=q$ for all $q \in Q \backslash Q_{1}$. Indeed, assume $q=p_{\beta}$ and $f(q)=p_{\gamma}$, and then, by the choice of $Q_{1}, \beta, \gamma>\alpha$, and we shall show that $\gamma \neq \beta$ is impossible.

Case (i). $\beta>\gamma$ : In the $\gamma$ th step, $p_{\gamma}$ was chosen to be a point where $G_{\alpha}$ is a one-one function (i.e. $\left.p_{\gamma} \notin A_{\alpha}\right)$. In particular, there is at most one $x$ so that $\left(x, p_{\gamma}\right) \in G_{\alpha}$. In the $\beta$ th step, $p_{\beta}$ was chosen to be a point different from this $x$. Thus $\left(p_{\beta}, p_{\gamma}\right) \notin G_{\alpha}$, and $f\left(p_{\beta}\right) \neq p_{\gamma}$.

Case (ii). $\beta<\gamma$ : In the $\beta$ th step, $p_{\beta}$ was chosen in the complement of $A_{\alpha}$ so that there is at most one $x$ satisfying $\left(p_{\beta}, x\right) \in G_{\alpha}$. In the $\gamma$ th step, $p_{\gamma}$ was chosen to be different from this $x$, so again $\left(p_{\beta}, p_{\gamma}\right) \notin G_{\alpha}$ and $f\left(p_{\beta}\right) \neq p_{\gamma}$.

Proof of Lemma 3 . Let $P$ be the set constructed in Lemma 8 , and let $\left\{P_{\alpha}^{\prime}\right.$ : $\left.\alpha<\omega_{1}\right\}$ be a partition of $P$ to $\omega_{1}$ disjoint uncountable sets. By discarding at most countably many points from each $P_{\alpha}^{\prime}$, we obtain a subset $P_{\alpha}$ of $P_{\alpha}^{\prime}$ so that for each $a<b, P \cap[a, b)$ is uncountable whenever it is nonempty. We now put $K_{\alpha}=K\left(P_{\alpha}\right)$.

Proof of (i). Since $S$ is a retract of $K_{\alpha}$, we can assume (replacing $\varphi$ by $\varphi \circ r$, where $r: K_{\alpha} \rightarrow S$ is the retraction) that $S=K_{\alpha}$. Similarly, by retracting in $K_{\beta}$, we can assume that $\varphi\left(K_{\alpha}\right)$ is a clopen subset of $K_{\beta}$ determined by $[a, b) \subset P_{\beta}$. The map $\varphi$ induces an isomorphism, $\psi$, from the Boolean algebra of clopen subsets of $\varphi\left(K_{\alpha}\right)$ into $B\left(P_{\alpha}\right)$, and we consider the uncountable family of pairwise comparable elements $\left\{\psi[a, q): q \in[a, b) \cap P_{\beta}\right\}$ in $B\left(P_{\alpha}\right)$, and write each of them in the form $\psi[a, q)=\bigcup_{i=1}^{m(q)}\left[a_{2 i-1}(q), a_{2 i}(q)\right)$, with $a_{i}(q)<a_{i+1}(q)$ in $P_{\alpha}$. 
By passing to an uncountable subset $Q$ of $[a, b) \cap P_{\beta}$, we can assume

(1) $m(q)=m$ is constant for $q \in Q$;

(2) for each $i \leqslant 2 m$, the map $q \rightarrow a_{i}(q)$, for $q \in Q$ is either constant or one-one;

(3) for each $1 \leqslant i \leqslant m$ there is a rational number $r_{i}$, with $a_{2 i-1}(q)<r_{i}<a_{2 i}(q)$ for all $q \in Q$.

Let now $j \leqslant 2 m$ be the first index so that $q \rightarrow a_{j}(q)$ is one-one (such a $j$ exists because $\psi$ is one-one), and assume, say, that $j=2 k$ is even. Since the $k$ th intervals of $\psi[a, q)$ contain the fixed point $r_{k}$ for all $q \in Q$, and since $q>q_{1}$ implies that $\psi[a, q) \supset \psi\left[a, q_{1}\right)$, we must have that $a_{2 k}(q)>a_{2 k}\left(q_{1}\right)$ for all $q>q_{1}$ in $Q$, i.e. $q \rightarrow a_{j}(q)$ is strictly increasing on $Q$. Similarly, if $j$ is odd, $q \rightarrow a_{j}(q)$ is strictly decreasing on $Q$.

Thus we have found a strictly monotone map from an uncountable subset $Q \subset P_{\beta} \subset P$ into $P_{\alpha} \subset P$. Since $P_{\alpha} \cap P_{\beta}=\varnothing$ when $\alpha \neq \beta$, the construction of $P$ implies that $\alpha=\beta$.

Proof of (ii). It is clear that each $K_{\alpha}$ is CCC: Each disjoint family of clopen subsets in $K_{\alpha}$ induces a disjoint family of intervals in $\mathbf{R}$, hence is countable. Since the closed sets of $K_{\alpha}$ are retracts of $K_{\alpha}$, this is inherited to all closed subsets of $K_{\alpha}$.

Proof of (iii). Every open subset of $K_{\alpha}$, contains a nonempty clopen subset, determined by an interval $[a, b)$ in $P_{\alpha}$. Since such intervals, if nonempty, are uncountable, it follows that every clopen subset of $K_{\alpha}$ contains uncountably many different clopen subsets, and hence is nonmetrizable.

\section{REFERENCES}

1. Y. Benyamini, Constants of simultaneous extension of continuous functions, Israel J. Math. 16 (1973), $258-262$.

2. Small into-isomorphisms between spaces of continuous functions, Proc. Amer. Math. Soc. 83 (1981), 479-485.

3. Y. Benyamini, M. E. Rudin and M. Wage, Continuous images of weakly compact subsets of Banach spaces, Pacific J. Math. 70 (1977), 309-324.

4. E. Bishop and $\mathrm{K}$. de-Leeuw, The representation of linear functionals by measures on sets of extreme points, Ann. Inst. Fourier (Grenoble) 9 (1959), 305-331.

5. R. Bonnet, Very strongly rigid Boolean algebras continuum discrete set condition countable antichain condition. I, Algebra Universalis 11 (1980), 341-364.

6. P. R. Halmos, Lectures on Boolean algebras, Van Nostrand, Princeton, N. J., 1963.

7. I. Juhász, Cardinal functions in topology, Math. Centre Tracts, No. 34, Math. Centrum, Amsterdam, 1971.

8. A. Pelczynski, On $C(S)$ subspaces of separable Banach spaces, Studia Math. 31 (1968), 513-522.

9. W. Sierpinski, Sur les type d'ordre des ensembles linéaire, Fund. Math. 37 (1950), 253-264.

Department of Mathematics, Technion-Israel Institute of Technology, Haifa 32000, IsRael 